\title{
Quantitative Analysis on an Electron Probe with the NIST Transition Edge Sensor Microcalorimeter X-ray Detector
}

\author{
Terrence Jach, ${ }^{*}$ Joel Ullom, ${ }^{* *}$ Nicholas Ritchie, ${ }^{*}$ James Beall**
}

* Chemical Science and Technology Laboratory, National Institute of Standards and Technology, MS8371, Gaithersburg, MD 20899-8371

**Electronics and Electrical Engineering Laboratory, National Institute of Standards and Technology, Boulder, CO 80303

The NIST microcalorimeter $\mathrm{x}$-ray detector is a versatile instrument that combines the high resolution of wavelength-dispersive detectors with the extended range of energy-dispersive detectors. The detector is a small calorimeter operated at a low temperature $(70 \mathrm{mK})$. The temperature change caused by the absorption of single x-ray photons is registered by a current through the transition edge sensor (TES) - a thin metal film that undergoes a superconducting-normal transition at the operating point. Our present detector has demonstrated a FWHM resolution of $4.4 \mathrm{eV}$ at $5.9 \mathrm{keV}$. The technology of microcalorimeters is more complicated than most x-ray detectors, so that a new set of operating considerations has emerged in the process of developing it as an analytical instrument. In particular, improvements in thermal and electrical stability will be described.

We report here the first operation of the NIST TES microcalorimeter x-ray detector to perform quantitative analysis of a sample with an electron probe. The detector element is mounted at the end of a probe projecting from the dewar into the electron microscope. The probe extends horizontally, which requires a sample mounting angle of $45^{\circ}$ to optimize the yield with a vertical electron beam. The sample was analyzed on a microbeam probe with a beam current of $7 \mathrm{nA}$ at an energy of 12 $\mathrm{keV}$.

The detector is a microcalorimeter element $400 \mu \mathrm{m}$ square, located approximately $30 \mathrm{~mm}$ from the source of the radiation. Its energy range is $400 \mathrm{eV}-7 \mathrm{keV}$. Count rates of 50/s $-170 / \mathrm{s}$ were registered while taking spectra of the sample and related standards. Spectra were processed using conventional analog pulse amplifiers and pulse-pileup circuitry modified for the relatively long period of the microcalorimeter pulses. The pulses were recorded with a $16 \mathrm{~K}$ channel multichannel analyzer board with an approximate resolution of $0.5 \mathrm{eV} /$ channel. All spectra were obtained in $1000 \mathrm{~s}$ live time.

The sample was the K-411 glass which is part of the NIST Standard Reference Material 470, Mineral Glasses for Microanalysis, a standard intended for use with electron probe microanalysis and SIMS. It contains previously analyzed quantities of $\mathrm{SiO}_{2}, \mathrm{MgO}, \mathrm{FeO}$, and $\mathrm{CaO}$. Spectra were also taken of reference standards consisting of $\mathrm{Fe}, \mathrm{SiO}_{2}, \mathrm{MgO}$, and chloroapatite $\left[\mathrm{Ca}_{5}\left(\mathrm{PO}_{4}\right)_{3} \mathrm{Cl}\right]$. A spectrum of K-411 glass used in the quantitative analysis is shown in Fig. 1.

Calculations were carried out using a Monte Carlo procedure which varied the composition of the respective elements. The analysis will be discussed in detail. The maximum deviation from the concentrations certified in the NIST SRM in the first round of analysis is $2 \%$. The work demonstrates that the NIST TES microcalorimeter detector has considerable potential for the analysis of x-ray fluorescence lines which would overlap in current energy-dispersive detectors or be too low in energy for most wavelength-dispersive detectors. 


\section{K-411 spectrum}

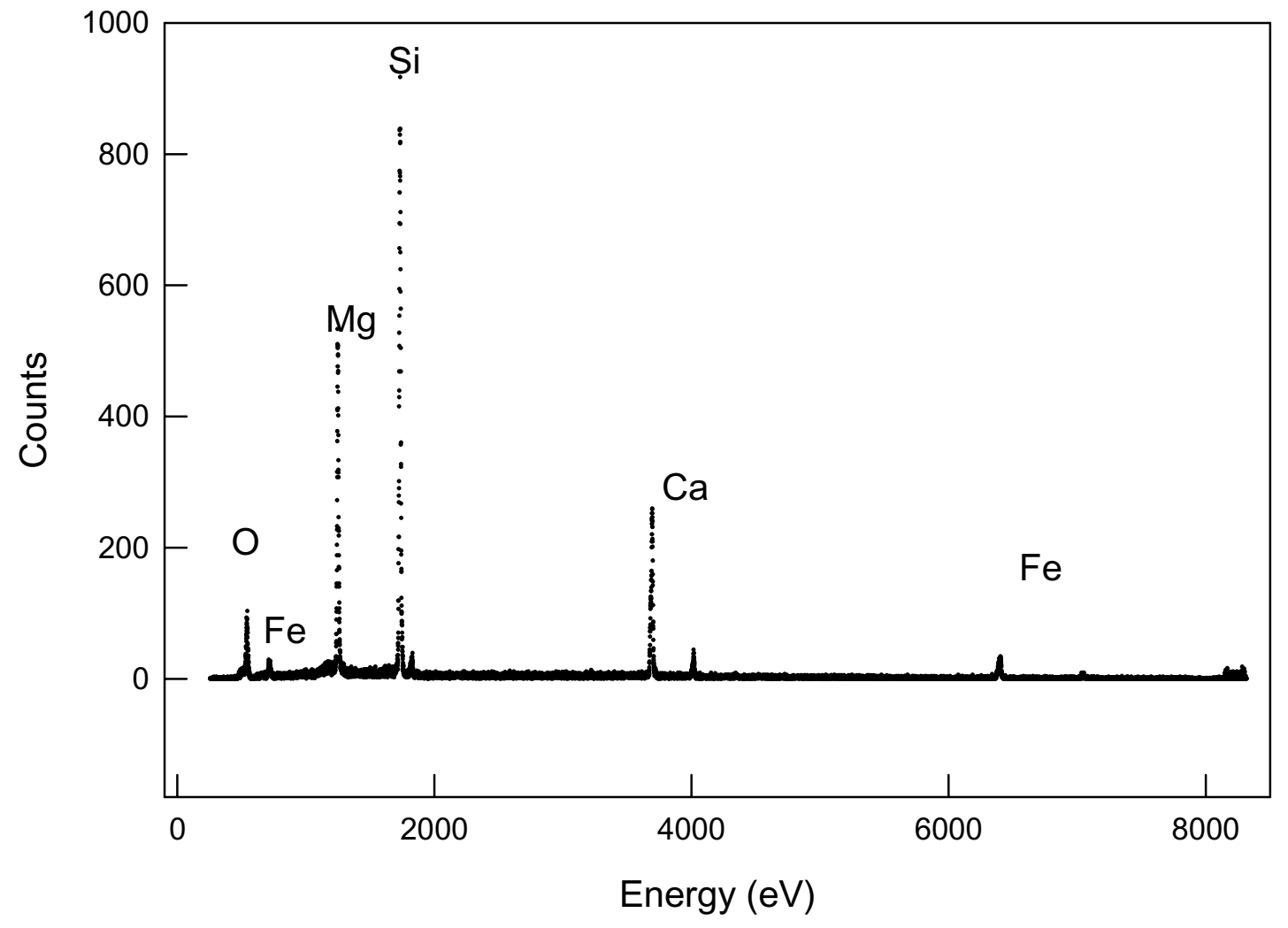

Fig. 1 Spectrum of the K-411 Standard Reference Material glass taken with the NIST TES microcalorimeter $\mathrm{x}$-ray detector excited by an electron beam. 